\title{
Article \\ Non-TZF Transcriptional Activator AtC3H12 Negatively Affects Seed Germination and Seedling Development in Arabidopsis
}

\author{
Hye-Yeon Seok ${ }^{1,+}$, Taehyoung Kim ${ }^{2,+}$, Sun-Young Lee ${ }^{1}$ and Yong-Hwan Moon ${ }^{1,2,3, * \mathbb{D}}$ \\ 1 Institute of Systems Biology, Pusan National University, Busan 46241, Korea; seokhyeon@pusan.ac.kr (H.-Y.S.); \\ symoonlee@pusan.ac.kr (S.-Y.L.) \\ 2 Department of Integrated Biological Science, Pusan National University, Busan 46241, Korea; \\ kth3245@naver.com \\ 3 Department of Molecular Biology, Pusan National University, Busan 46241, Korea \\ * Correspondence: moonyh@pusan.ac.kr; Tel.: +82-51-510-2592 \\ + These authors contributed equally in this work.
}

check for updates

Citation: Seok, H.-Y.; Kim, T.; Lee, S.-Y.; Moon, Y.-H. Non-TZF Transcriptional Activator AtC3H12 Negatively Affects Seed Germination and Seedling Development in Arabidopsis. Int. J. Mol. Sci. 2022, 23, 1572. https://doi.org/10.3390/ ijms23031572

Received: 20 December 2021

Accepted: 28 January 2022

Published: 29 January 2022

Publisher's Note: MDPI stays neutral with regard to jurisdictional claims in published maps and institutional affiliations.

Copyright: (C) 2022 by the authors. Licensee MDPI, Basel, Switzerland. This article is an open access article distributed under the terms and conditions of the Creative Commons Attribution (CC BY) license (https:// creativecommons.org/licenses/by/ $4.0 /)$.

\begin{abstract}
CCCH}$ zinc finger proteins are a large protein family and are classified as either tandem $\mathrm{CCCH}$ zinc finger (TZF) or non-TZF proteins. The roles of TZF genes in several plants have been well determined, whereas the functions of many non-TZF genes in plants remain uncharacterized. Herein, we describe biological and molecular functions of AtC3H12, an Arabidopsis non-TZF protein containing three $\mathrm{CCCH}$ zinc finger motifs. AtC3H12 has orthologs in several plant species but has no paralog in Arabidopsis. AtC3H12-overexpressing transgenic plants (OXs) germinated slower than wild-type (WT) plants, whereas atc3h12 mutants germinated faster than WT plants. The fresh weight (FW) and primary root lengths of AtC3H12 OX seedlings were lighter and shorter than those of WT seedlings, respectively. In contrast, FW and primary root lengths of atc $3 h 12$ seedlings were heavier and longer than those of WT seedlings, respectively. AtC3H12 was localized in the nucleus and displayed transactivation activity in both yeast and Arabidopsis. We found that the 97-197 aa region of AtC3H12 is an important part for its transactivation activity. Detection of expression levels and analysis of Arabidopsis transgenic plants harboring a $P_{\mathrm{AtC} 3 \mathrm{H} 12}:$ GUS construct showed that AtC3H12 expression increases as the Arabidopsis seedlings develop. Taken together, our results demonstrate that $\mathrm{AtC} 3 \mathrm{H} 12$ negatively affects seed germination and seedling development as a nuclear transcriptional activator in Arabidopsis. To our knowledge, this is the first report to show that non-TZF proteins negatively affect plant development as nuclear transcriptional activators.
\end{abstract}

Keywords: Arabidopsis; AtC3H12; CCCH zinc finger; non-TZF; seed germination; seedling development; transcriptional activator

\section{Introduction}

Zinc finger proteins constitute a large group of protein families categorized to different types, such as $\mathrm{C} 2 \mathrm{H} 2, \mathrm{C} 2 \mathrm{C} 2, \mathrm{C} 2 \mathrm{HC}, \mathrm{C} 2 \mathrm{C} 2 \mathrm{C} 2 \mathrm{C} 2, \mathrm{C} 2 \mathrm{HCC} 2 \mathrm{C} 2$, and $\mathrm{CCCH}$, on the basis of the number and order of cysteine (Cys) and histidine (His) residues that bind to a zinc ion [1,2]. They participate in various biological processes, including transcription, apoptosis, and protein assembly [1,3,4].

$\mathrm{CCCH}$ zinc finger proteins, which are broadly found in yeast and higher eukaryotes, are determined based on the $\mathrm{CCCH}$ zinc finger motif, which consists of three Cys residues and one subsequent His residue [5]. Sixty-eight $\mathrm{CCCH}$ zinc finger protein genes have been recognized in the Arabidopsis (Arabidopsis thaliana) genome, whereas 67 genes have been recognized in rice (Oryza sativa) [5]. Of the $68 \mathrm{CCCH}$ zinc finger proteins in Arabidopsis, 26 are tandem $\mathrm{CCCH}$ zinc finger (TZF) proteins with two tandem $\mathrm{CCCH}$ zinc finger motifs, and 42 are non-TZF proteins with one or more than two CCCH zinc finger motifs [6].

To date, the functions of TZF genes have been well studied in several plants. In Arabidopsis, AtTZF3/Oxidation-related Zinc Finger 2 (AtOZF2) is engaged in abscisic acid 
(ABA) response and the salt stress response [7]. AtTZF4/SOMNUS (SOM) negatively regulates seed germination by regulating $\mathrm{ABA}$ and gibberellic acid (GA) metabolic genes [8]. AtTZF6 / PEI1 plays an important role during embryogenesis [9]. AtTZF9 mediates immune response triggered by pathogen-associated molecular patterns [10]. AtTZF10/Salt-inducible Zinc Finger 2 (AtSZF2) and AtTZF11/AtSZF1 function in the salt stress response [11]. In rice, OsTZF1 confers abiotic stress tolerance and negatively modulates leaf senescence under stress conditions [12]. OsC $3 \mathrm{H} 10$ modulates the expression of stress-responsive genes and improves drought tolerance [13]. PvC3H72, a TZF gene in switchgrass (Panicum vigatum), is involved in cold tolerance by controlling the ICE1-CBF-COR regulon and ABA-responsive genes [14]. Overexpression of PeC3H74, a TZF gene in moso bamboo (Phyllostachys edulis), in Arabidopsis renders the transgenic plants drought tolerant [15]. Similarly, overexpression of PdC3H17 in poplar (Populus deltoides $\times$ P. euramericana) enhances drought tolerance [16].

In contrast, the functional roles of non-TZF genes have not been well studied compared to TZF genes. In Arabidopsis, KHZ1/AtC3H36 and KHZ2 / AtC3H52 act not only in stress responses and aging but also in flowering [17]. AtC3H17 pleiotropically modulates development and the salt stress response [6,18]. In rice, the leaf and tiller angle increased controller $(O s L I C)$ is known to participate in architecture regulation mediated by brassinosteroid signaling [19]. IbC3H18, a non-TZF gene in sweet potato (Ipomoea batatas), is associated with abiotic stress tolerance [20]. Male Fertility 30a (BcMF30a) and BcMF30c in Chinese cabbage (Brassica campestris) are involved in pollen development [21,22]. AtC3H59/ZFWD3 interacting with deSUMOylating isopeptidase1 (Desi1) participates in seed germination, seedling development, and seed development [23]. Despite an increasing number of studies on non-TZF genes, the roles of many non-TZF genes in plants still remain unclear.

$\mathrm{CCCH}$ zinc finger proteins are known as RNA-binding proteins and associated with post-transcriptional regulation of mRNA. In Arabidopsis, AtTZF1, AtC3H14, AtC3H15/ AtCDM1, cleavage and polyadenylation specificity factor 30 (AtCPSF30), and HUA1 have been revealed to bind to RNA [4,24-26]. The TZF motif of AtTZF1 plays a key role in binding to RNA [25]. AtC3H3 possesses ribonuclease function, binding to RNA [27].

In addition to its role in RNA metabolism, most recent studies demonstrated that $\mathrm{CCCH}$ zinc finger proteins also modulate transcription. Two Arabidopsis TZF proteins, AtC3H14 and AtC3H15/AtCDM1, display transactivation activity and bind to both DNA and RNA in vitro [4]. OsLIC activates transcription via its EELR domain [19]. Recently, AtC3H17, PdC3H17, IbC3H18, and $\mathrm{PvC} 3 \mathrm{H} 72$ have also been revealed to be transcriptional activators $[6,14,16,20]$.

Well-conserved transactivation motifs have been recognized on transcriptional activators in plants. In CCCH zinc finger proteins, the EELR motif in OsLIC has been identified as a transactivation motif [19]. Similarly, the EELR-like motif of AtC3H17 is responsible for transactivation activity [6]. The AHA motif in the homeodomain-leucine zipper (HD-Zip) I family proteins, including Homeobox 1 (AtHB1), AtHB7, AtBH12, and AtHB13, also functions in transcriptional activation [28]. Additionally, the LWSY and EDLL motifs serve as transactivation motifs in Apetala 2 (AP2)/ ethylene-responsive factor (ERF) transcription factors, such as related to AP2.12 (RAP2.12), RAP2.2, and AtERF98 [29,30]. Recently, EELL-, VDDG-, and LWSY-like motifs in AtERF73/hypoxia-responsive ERF 1 (HRE1), an Arabidopsis AP2/ERF transcription factor, have been reported as transactivation motifs [31].

In this study, we selected a previously uncharacterized Arabidopsis non-TZF protein, AtC3H12 showing strong transactivation activity and characterized its biological and molecular functions. We demonstrated that $\mathrm{AtC} 3 \mathrm{H} 12$ negatively affects seed germination and seedling development as a nuclear transcriptional activator. Our findings will enable us to expand our knowledge of the functions of non-TZF proteins as transcriptional regulators.

\section{Results}

\subsection{AtC3H12 Has Three CCCH Zinc Finger Motifs}

To isolate non-TZF protein(s) that act as transcription factors, we screened non-TZF gene(s) that show high transactivation activity in yeast and selected AtC3H12 (At1g32360) 
for further study (data not shown). AtC3H12, a non-TZF protein, has three CCCH zinc finger motifs that are designated as $C-X_{8}-C-X_{5}-C-X_{3}-H$ (Figure $\left.1 a\right)$. In order to identify homologous genes of AtC3H12, BLASTP analysis was performed. AtC3H12 has no paralog in Arabidopsis but has several orthologs in other plant species, such as Arabidopsis lyrata, Camelina sativa, Capsella rubella, Eutrema salsugineum, Brassica napus, Brassica rapa, and Brassica oleracea (data not shown). Multiple-sequence alignment showed that amino acid sequences were highly conserved among AtC3H12 and its orthologs, especially in the N-terminus, CCCH zinc finger motifs, and C-terminus (Figure 1b).

(a)

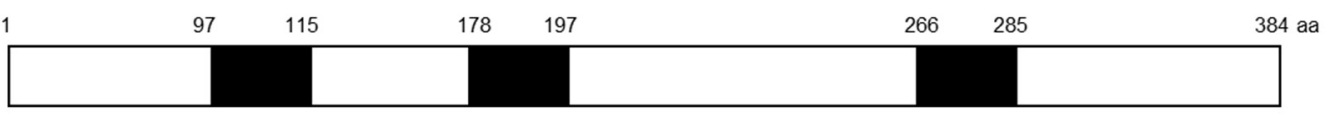

$\mathrm{CCCH}$ zinc finger motif $\left(\mathrm{C}-\mathrm{X}_{8}-\mathrm{C}-\mathrm{X}_{5}-\mathrm{C}-\mathrm{X}_{3}-\mathrm{H}\right)$

(b)

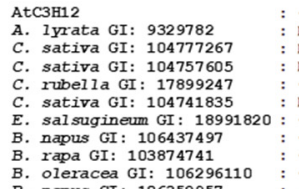
B. Oleracea GI: 106296110
B. napus GI : 106352957

Atc3H12
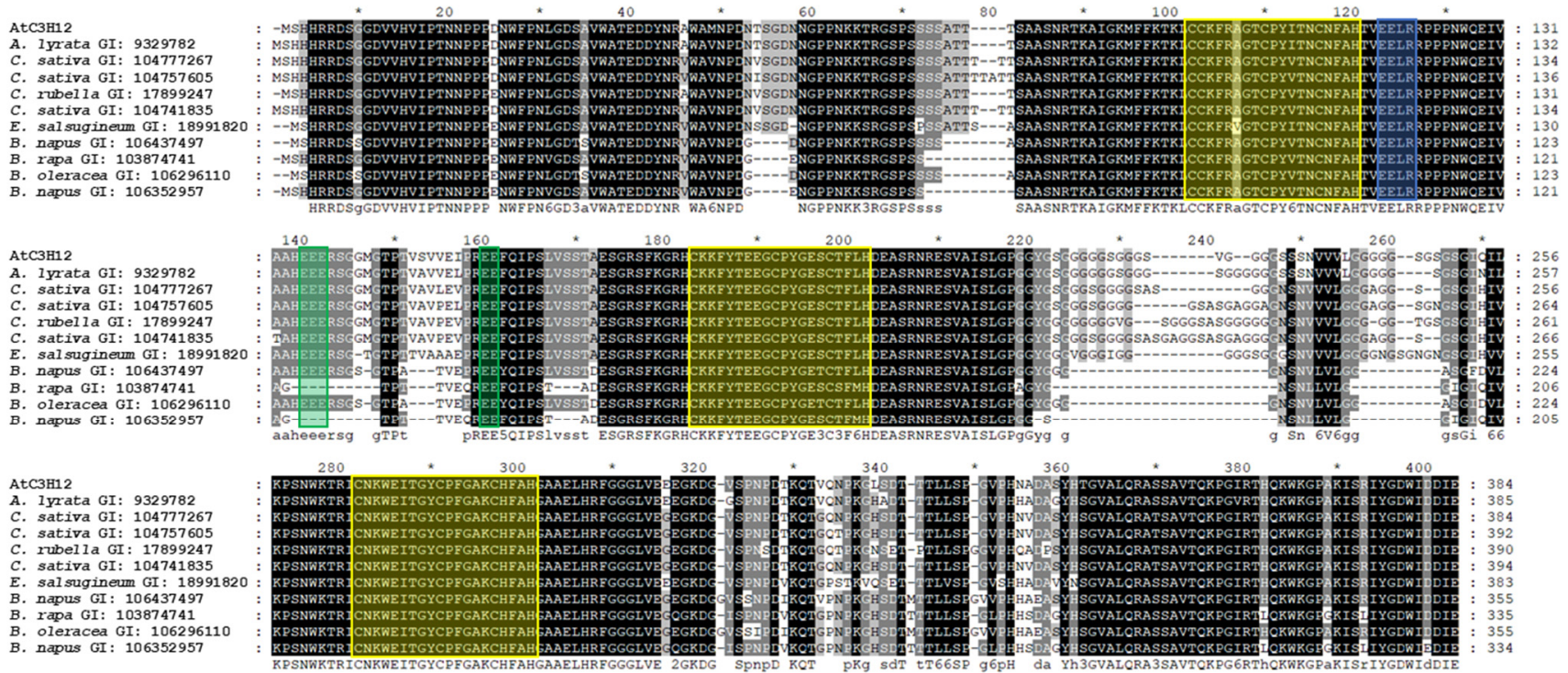

$\mathrm{CCCH}$ zinc finger motif

EELR motif

Conserved Glu residues

Figure 1. Protein domain of AtC3H12 and multiple alignment among AtC3H12 and its orthologs. (a) $\mathrm{CCCH}$ zinc finger motifs of $\mathrm{AtC} 3 \mathrm{H} 12$. $\mathrm{CCCH}$ zinc finger motifs are represented as black boxes. (b) Multiple-sequence alignment was performed using the ClustalW2 program with amino acid sequences of AtC3H12 and its orthologs. Three conserved $\mathrm{CCCH}$ zinc finger motifs are annotated as yellow boxes. Black-, dark-gray-, and light-gray-shaded amino acids represent $100 \%, 80 \%$, and $60 \%$ conservation rate, respectively. Blue and green boxes represent the EELR motif and conserved Glu residues, respectively. * display positions which have a single, fully conserved residue. The GI number of each protein sequence is as follows: AtC3H12, 840128; A. lyrata, 9329782; C. sativa, 104777267; C. sativa, 104757605; C. rubella, 17899247; C. sativa, 104741835; E. salsugineum, 18991820; B. napus, 106437497; B. rapa, 103874741; B. oleracea, 106296110; B. napus, 106352957.

\subsection{AtC3H12 Is Negatively Associated with Seed Germination and Seedling Development}

To study the biological functions of AtC3H12, we generated AtC3H12-overexpressing transgenic plants (OXs) and selected homozygous atc3h12 mutants (Figures S1 and S2). First, seed germination was analyzed. Seeds of AtC3H12 OX plants germinated significantly slower than those of WT plants, whereas atc3h12 mutants germinated faster than WT (Figure 2a,b and S3a,b). In particular, the germination percentage of AtC3H12 OXs and atc3h12 mutants was significantly lower and higher, respectively, than that of the WT 2 days after germination (DAG) (Figure $2 b$ and $\mathrm{S} 3 b$ ). However, the final germination percentage did not differ among AtC3H12 OXs, atc3h12 mutants, and WT (Figure 2b and S3b). During seedling development from 7 to 18 DAG, AtC3H12 OX seedlings were smaller and lighter 
than WT seedlings (Figure 2c,d and S3c,d). In contrast, atc3h12 seedlings were heavier and larger than WT seedlings (Figure 2c,d). In particular, the increase in fresh weight (FW) from 10 to 14 DAG in atc3h12 mutants was higher than that of WT (Figure 2d). In addition, we found that the primary root length of AtC3H12 OXs was shorter than that of WT, whereas the primary root length of atc3h12 mutants was longer than that of WT from 7 to 18 DAG (Figure 2e,f and S3e,f). These results indicate that $A t C 3 H 12$ has a negative role in seed germination and seedling development.

(a)

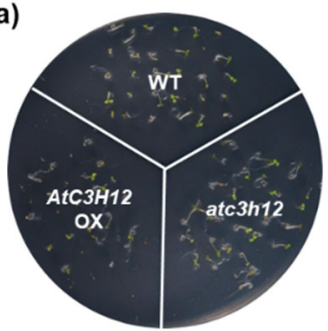

(c)

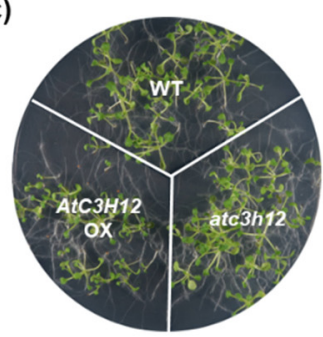

(e)

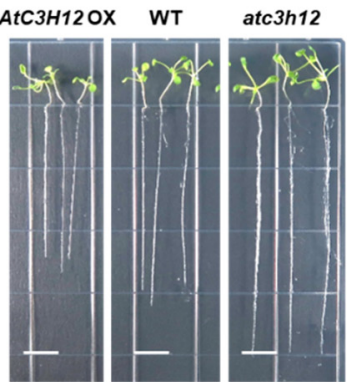

(b)

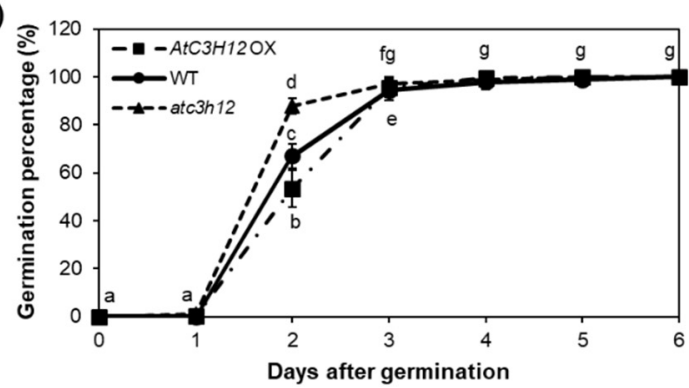

(d)

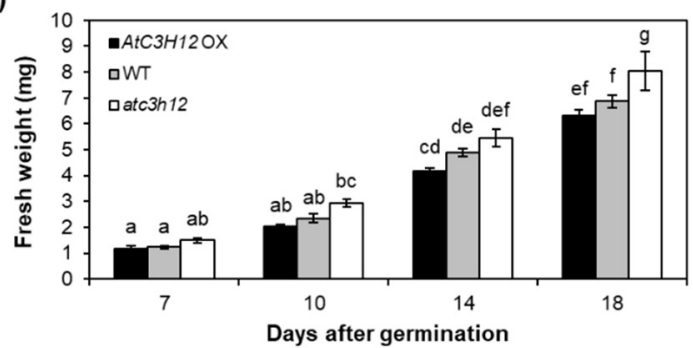

(f)

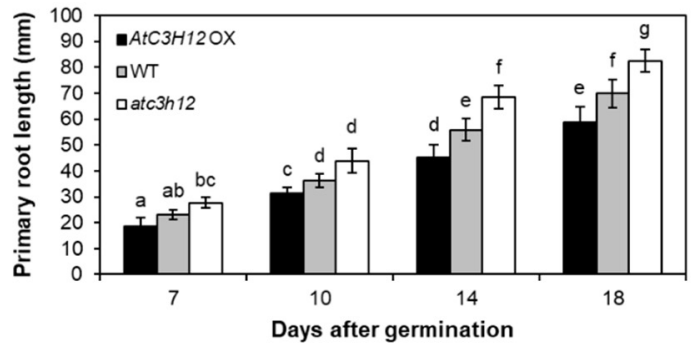

Figure 2. Seed germination and seedling development of AtC3H12 OXs and atc3h12 mutants. (a) Four-day-old WT, AtC3H12 OX-7, and atc3h12 seedlings grown on Murashige and Skoog (MS) agar plates under short-day (SD) conditions. (b) Germination percentage of WT, AtC3H12 OX-7, and atc $3 h 12$ mutants measured at specified times after sowing on MS agar plates. Germination was verified by radicle protrusion. Error bars display standard deviation $(n=20)$. (c) Fourteen-day-old WT, AtC3H12 OX-7, and atc3h12 seedlings grown on MS agar plates under SD conditions. (d) Fresh weight of WT, AtC3H12 OX-7, and atc3h12 seedlings grown on MS agar plates at 7, 10, 14, and 18 DAG. Error bars display standard deviation $(n=5)$. (e) Elongation of primary roots of WT, AtC3H12 OX-7, and atc3h12 seedlings at 14 DAG. The white lines indicate scale bar $=1 \mathrm{~cm}$. (f) Primary root lengths of WT, AtC3H12 OX-7, and atc3h12 seedlings grown on MS agar plates under SD conditions were measured at 7, 10, 14, and 18 DAG. Error bars display standard deviation $(n=10)$. In $(\mathbf{b}, \mathbf{d}, \mathbf{f})$, different letters display significant differences $(p<0.05)$.

We investigated the flowering time of AtC3H12 OXs and atc3h12 mutants to determine whether $A t C 3 H 12$ functions at the reproductive developmental stage as well as at the vegetative developmental stage. To determine flowering time, the number of rosette leaves was counted at bolting under long-day conditions. There was no considerable difference in 
flowering time among WT, AtC3H12 OXs, and atc3h12 mutants (Figure S4), indicating that AtC3H12 may not be engaged in the regulation of flowering time.

\subsection{AtC3H12 Protein Is Localized in the Nucleus}

We studied the subcellular localization of the AtC3H12 protein in Arabidopsis protoplasts using N-terminal (sGFP-AtC3H12) and C-terminal (AtC3H12-sGFP) synthetic green fluorescent protein (sGFP)-fused AtC3H12 constructs to determine the potential molecular function of the protein (Figure 3a). As a result, GFP signals of sGFP-AtC3H12 and AtC3H12-sGFP constructs were exclusively observed in the nucleus (Figure $3 b$ ), indicating that $\mathrm{AtC} 3 \mathrm{H} 12$ may exert its functions in the nucleus.

(a)

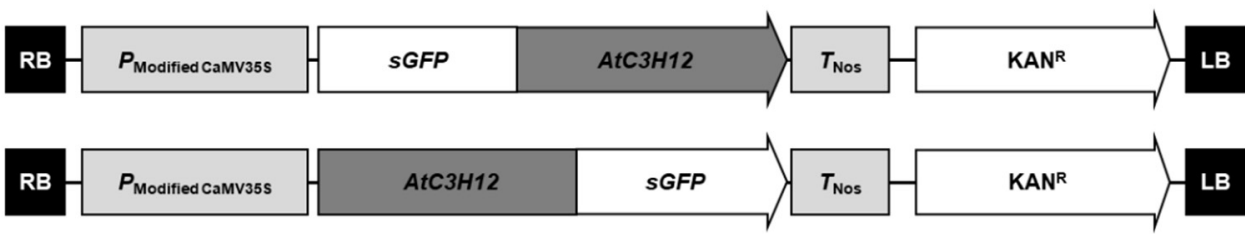

(b)

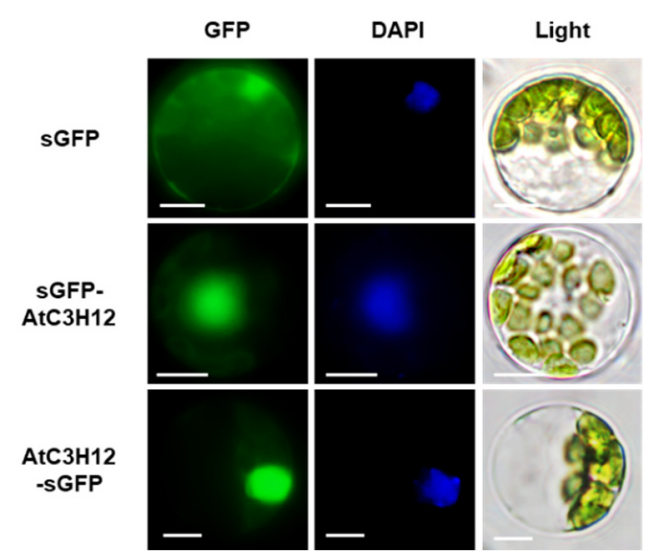

Figure 3. Subcellular localization of AtC3H12. (a) Schematic maps of the sGFP-fused, full-length ORF of AtC3H12 constructs. (b) Subcellular localization of AtC3H12 protein investigated by transient expression of sGFP-AtC3H12 and AtC3H12-sGFP constructs in Arabidopsis protoplasts. Left, GFP signal; middle, $4^{\prime}$,6-diamidino-2-phenylindole (DAPI) staining; right, light microscopic picture. The white lines indicate scale bar $=10 \mu \mathrm{m}$.

\subsection{The 97-197 aa Region of AtC3H12 Is Responsible for Its Transactivation Activity}

To determine the transactivation domain of $\mathrm{AtC} 3 \mathrm{H} 12, \mathrm{AtC} 3 \mathrm{H} 12$ was divided into two regions: the N-terminal 1-197 aa region (N197), in which the first and the second $\mathrm{CCCH}$ zinc finger motifs were contained, and the C-terminal 178-384 aa region (C207), in which the second and third CCCH zinc finger motifs were present (Figure $4 \mathrm{~b}$ ). The full-length open reading frame (ORF), N197, and C207 were separately cloned into pBD-GAL4 to generate GAL4 DNA-binding domain (BD)-AtC3H12 fusion constructs (Figure 4a) and transformed into yeast. In a quantitative $\beta$-galactosidase orthonitrophenyl- $\beta$-D-galactopyranoside (ONPG) assay and yeast growth assay, N197 showed transactivation activity in yeast as well as full-length ORF (Figure 4c,d and S5a).

To narrow down the transactivation domain, N197 of AtC3H12 was divided into two regions, the 1-115 aa region (NN115) and 97-197 aa region (NC101), and cloned into pBD-GAL4 (Figure 5a,b). In the ONPG assay and yeast growth assay using the yeast transformants containing GAL4 BD-AtC3H12 fusion constructs, NC101 showed transactivation activity, whereas NN115 displayed no activity (Figure 5c,d and S5b). 
(a)

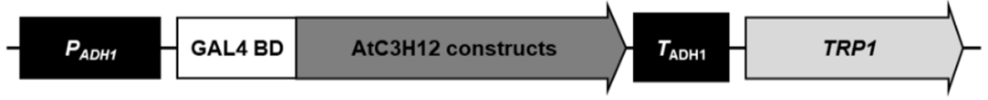

(b)

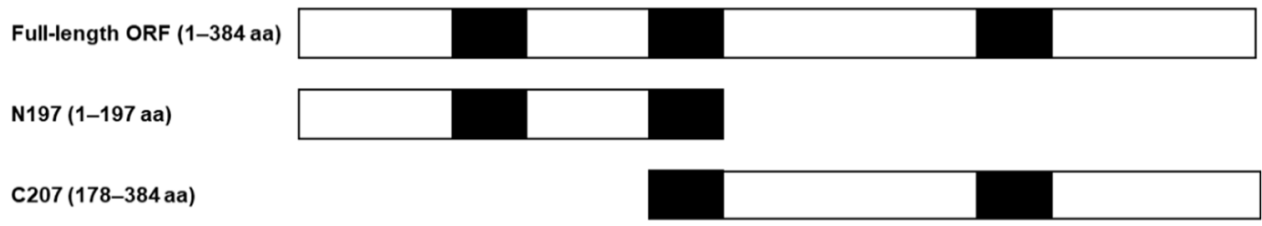

(c)

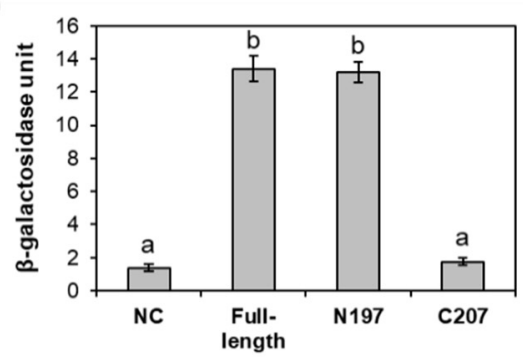

(d)

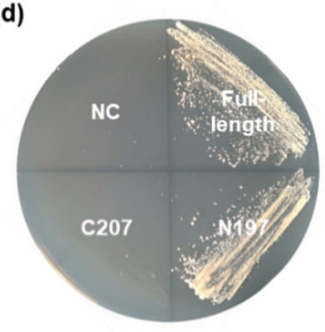

Figure 4. Transactivation activity assay of AtC3H12 in yeast. (a) Schematic map of the GAL4 BDfusion vector for transactivation activity assay in yeast. (b) Schematic maps of full-length ORF, N197, and C207 of AtC3H12 for transactivation activity assay. (c) Quantitative $\beta$-galactosidase ONPG assay. $\beta$-Galactosidase activities were measured to quantify the transactivation activities. Error bars display standard deviation $(n=3)$. Different letters display significant differences $(p<0.05)$. (d) Yeast growth assay. Yeast transformants were grown on SD media lacking Trp and Ura (SD-Trp/-Ura). In (c,d), empty pBD-GAL4 vector was used for a negative control. NC, negative control.

(a)

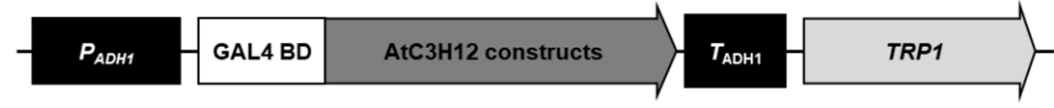

(b)

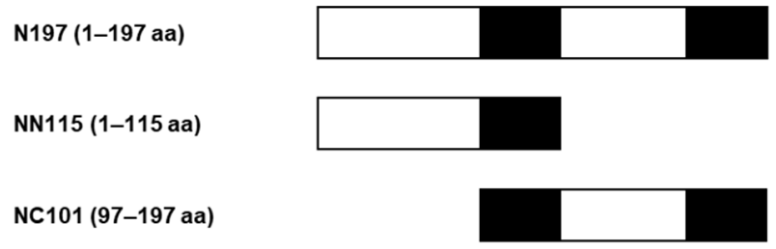

(c)

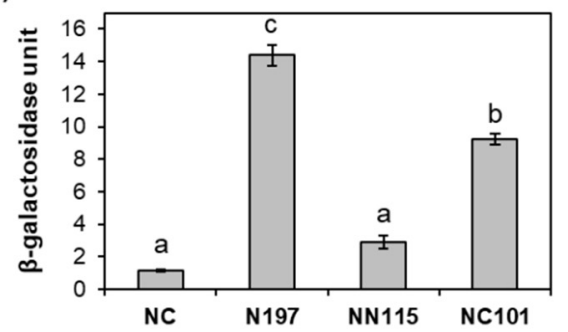

(d)

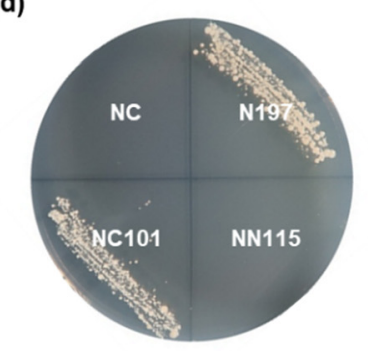

Figure 5. Transactivation activity assay of N197 of AtC3H12 in yeast. (a) Schematic map of the GAL4 BD-fusion vector for transactivation activity assay in yeast. (b) Schematic maps of N197, NN115, and NC101 of AtC3H12 for transactivation activity assay. (c) Quantitative $\beta$-galactosidase ONPG assay. $\beta$-Galactosidase activities were measured to quantify the transactivation activities. Error bars display standard deviation $(n=3)$. Different letters display significant differences $(p<0.05)$. (d) Yeast growth assay. Yeast transformants were grown on SD-Trp/-Ura. In (c,d), empty pBD-GAL4 vector was used for a negative control. NC, negative control. 
To verify the transactivation activity of AtC3H12 in Arabidopsis, effector vectors, in which the full-length ORF, N197, or NC101 of AtC3H12 were linked to GAL4 BD, were generated and introduced into Arabidopsis protoplasts (Figure 6a). Transient expression of each effector vector along with reporter vector demonstrated that all of the full-length ORF, N197, and NC101 showed transactivation activity (Figure 6b). These results are compatible with the data acquired from yeast (Figures $4 \mathrm{c}$ and 5c) and suggest that NC101 is responsible for the transactivation activity of AtC3H12.

(a)

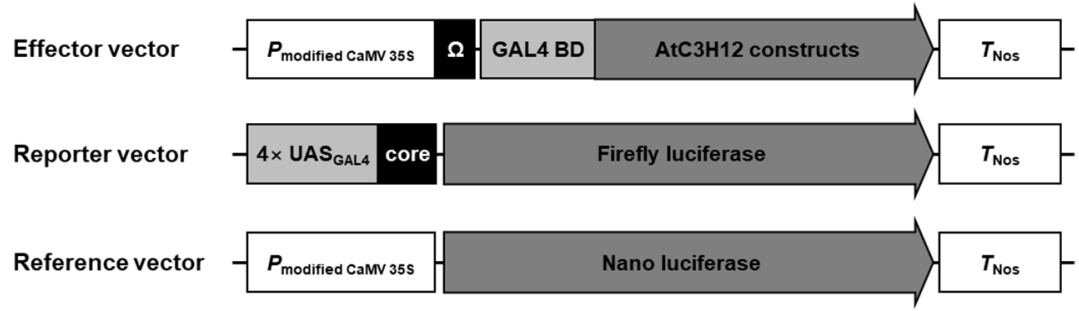

(b)

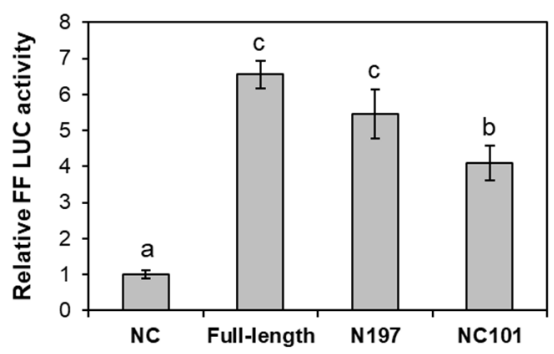

Figure 6. Transactivation activity assay of AtC3H12 in Arabidopsis protoplasts. (a) Schematic maps of the effector vector, reporter vector, and reference vector for transactivation activity assay. (b) Relative firefly luciferase (FF LUC) activities of full-length ORF, N197, and NC101 of AtC3H12 in Arabidopsis protoplasts. The reference vector was used for the normalization of transformation efficiency. The empty effector vector was used for a negative control. Normalized FF LUC activity of negative control was set to 1 . Error bars display standard deviation $(n=3)$. Different letters display significant differences $(p<0.05)$.

Next, we compared the amino acid sequences of NC101 of AtC3H12 and the corresponding regions of AtC3H12 orthologs. Amino acid sequences of the regions were highly conserved among the NC101 and orthologs, especially in the EELR motif and Glu residues (Figure 1b). It has previously been reported that the EELR motif is important for transactivation activity [19]. In addition, acidic amino acid residues, such as Glu and Asp, are also involved in transactivation activity [32]. These results suggest that the EELR motif and conserved Glu residues in NC101 of AtC3H12 might play a key role in its transactivation activity.

\subsection{Expression Levels of AtC3H12 during Development and in the Organs of Arabidopsis}

We examined the expression patterns of $A t C 3 H 12$ in different developmental stages and organs by quantitative RT-PCR (RT-qPCR). The AtC3H12 transcript level was slightly elevated as the seedlings developed from 4 to 21 days after germination (DAG) (Figure 7a). In mature plants, cauline leaves and rosette leaves showed a higher level of AtC3H12 transcripts than other organs, such as roots, stems, floral clusters, and siliques (Figure $7 \mathrm{~b}$ ). 
(a)

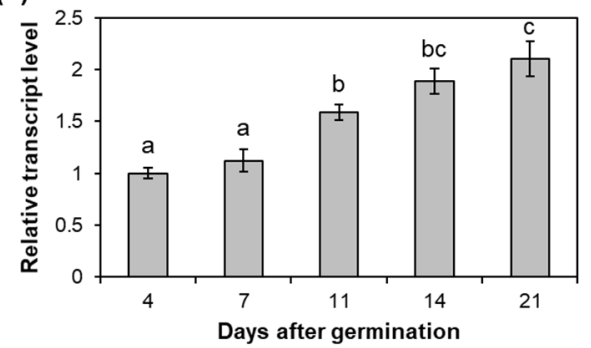

(b)

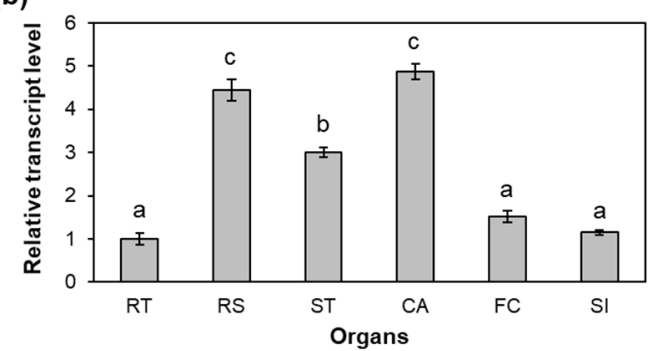

Figure 7. Temporal and spatial expression patterns of AtC3H12. (a) Quantitative RT-PCR (RT-qPCR) analysis of AtC3H12 in 4-, 7-, 11-, 14-, and 21-day-old WT seedlings grown under SD conditions. Transcript level in 4-day-old seedlings was set as 1. (b) RT-qPCR analysis of AtC3H12 in organs of 50-day-old WT plants grown under long-day conditions. Transcript level in RT was set as 1. RT, roots; $\mathrm{RS}$, rosette leaves; ST, stems; CA, cauline leaves; FC, floral clusters; SI, siliques. GAPC was used for an endogenous control gene. At least two biological replicates showed similar results. Error bars display standard deviation $(n=3)$. Different letters display significant differences $(p<0.05)$.

To visualize the expression patterns of $A t C 3 H 12$, transgenic plants harboring the

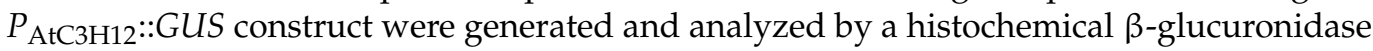
(GUS) assay (Figure 8a). First, we compared the promoter activities of AtC3H12 with and without the 559-bp $5^{\prime}$ UTR (Figure S6a). As a result, the AtC3H12 promoter with $5^{\prime}$ UTR showed insignificant GUS activity (Figure $\mathrm{S} 6 \mathrm{~b}$ ). Thus, we used $P_{\mathrm{AtC} 3 \mathrm{H} 12}:$ GUS transgenic plants without the $5^{\prime}$ UTR for further experiments. The histochemical GUS assay revealed that GUS activity was detected mainly in the cotyledons and the leaves of 7, 11, 14, and 21 DAG seedlings, and the activity increased as the seedlings grew (Figure $8 \mathrm{~b}$ ), supporting the result that $A t C 3 H 12$ expression increases as plants develop (Figure 7a).

(a)

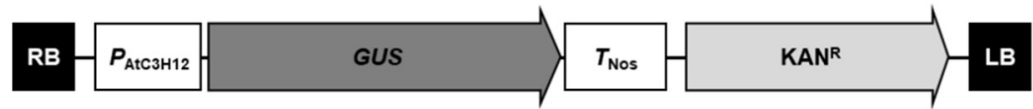

(b)
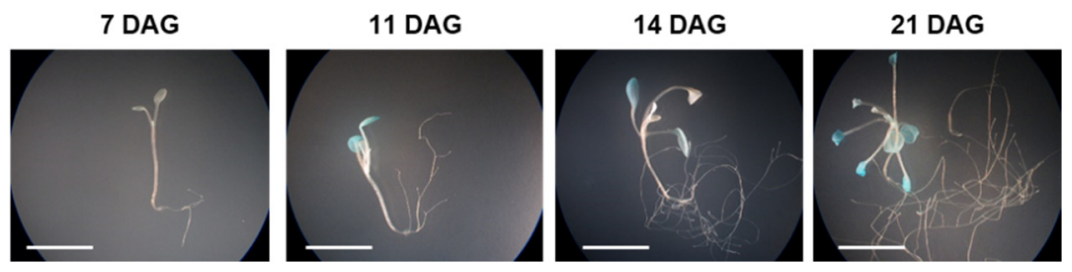

Figure 8. Analysis of the promoter activity of AtC3H12. (a) Schematic map of $P_{\mathrm{AtC} 3 \mathrm{H} 12:: G U S \text { for }}$ GUS assay. (b) Histochemical assay of GUS activities in transgenic plants harboring $P_{\mathrm{AtC} 3 \mathrm{H} 12}:: G U S$ at different developmental stages grown under SD conditions. Three independent $T_{1}$ lines showed similar results, with one shown here. The white lines indicate scale bar $=1 \mathrm{~cm}$.

\section{Discussion}

$\mathrm{CCCH}$ zinc finger proteins are classified into two groups, TZF and non-TZF proteins [6]. Although non-TZF genes have been recently studied in several plant species, many still remain uncharacterized. Herein, we studied the functions of the Arabidopsis non-TZF gene, AtC3H12.

AtC3H12 has three CCCH zinc finger motifs (Figure 1a). Our BLASTP analysis showed that AtC3H12 has orthologs in several plant species, but no paralog in Arabidopsis (Figure $1 b$ ), indicating that it is a unique gene in Arabidopsis. Our phenotype analysis using AtC3H12 OXs and atc3h12 mutants showed that AtC3H12 plays important roles in seed germination and seedling development (Figure 2 and S3). AtC3H12 was localized in the nucleus and showed transactivation activity via its 97-197 aa region (Figures 3-6), demon- 
strating that $\mathrm{AtC} 3 \mathrm{H} 12$ has pleiotropic effects during Arabidopsis vegetative development by transactivating downstream genes.

At the beginning of the study of the $\mathrm{CCCH}$ zinc finger proteins, the proteins were recognized as RNA-binding proteins participating in post-transcriptional regulation, including AtTZF1, AtC3H14, AtC3H15/AtCDM1, AtCPSF30, and HUA1 in Arabidopsis [4,24-26]. $\mathrm{CCCH}$ zinc finger proteins, such as AtC3H17, OsLIC, PdC3H17, IbC3H18, and $\mathrm{PvC} 3 \mathrm{H} 72$, have also been characterized as transcriptional regulators $[6,14,16,19,20]$. However, in spite of functional characterization studies of $\mathrm{CCCH}$ zinc finger proteins as transcriptional regulators, activation or repression motifs/domains have been identified in only limited CCCH zinc finger proteins. OsLIC has an EELR domain as a transactivation domain, and the EELR motif in the EELR domain has been well conserved among orthologs of OsLIC [19]. AtC3H17 has an EELR-like motif, which consists of EE(D/E)AL(K/R) [6]. Our study identified the 97-197 aa region of AtC3H12 as a transactivation domain (Figures 5 and 6). The EELR motif and Glu residues in the region are well conserved among AtC3H12 and its orthologs (Figure 1), showing that the EELR motif and Glu residues may play important roles in the transactivation activity of AtC3H12.

To reveal the biological function of AtC3H12 in Arabidopsis development, we generated AtC3H12 OX transgenic plants and obtained atc3h12 T-DNA-inserted mutants (Figures S1 and S2). Phenotypic analysis showed that AtC3H12 OXs germinated slower than WT, while atc3h12 mutants germinated faster than WT (Figure 2a,b and S3a,b). Moreover, AtC3H12 OX seedlings were smaller, and primary root length was shorter than WT seedlings, whereas atc $3 h 12$ seedlings were larger and primary root length was longer than WT seedlings (Figure $2 \mathrm{c}-\mathrm{f}$ and $\mathrm{S} 3 \mathrm{c}-\mathrm{f}$ ). These results suggest that $\mathrm{AtC} 3 \mathrm{H} 12$ negatively influences seed germination and seedling development in Arabidopsis. However, AtC3H12 OXs and atc $3 h 12$ mutants showed no significant differences in flowering time (Figure S4). This is the first report to show that a non-TZF protein negatively affects plant development as a nuclear transcriptional activator.

Several $\mathrm{CCCH}$ zinc finger genes participate in plant development in different ways. In Arabidopsis, overexpression of $A t C 3 H 17$ enhances seed germination, seedling development, and seed development [6], and AtC3H59/ZFWD3 also positively affects those processes, interacting with the PPPDE family protein Desi1 [23]. Similar to AtC3H12, overexpression of AtC3H14, an Arabidopsis TZF gene, resulted in defective cell elongation and dwarfism [33]. In rice, OsLIC is known to be involved in architecture regulation by the antagonistic function of Brassinazole-Resistant 1 (BZR1) [34]. These reports demonstrate that appropriate plant development is orchestrated by positive and negative developmental regulations. It is suggested that AtC3H12 might participate in the fine-tuning of development by negative regulation, together with other positive regulators in Arabidopsis. Recently, it has been reported that $A t C 3 H 12$ has a repressing effect on root hair density and root hair length depending on phosphorus availability [35]. It can be a clue to explain the function of AtC3H12. To explain how AtC3H12 negatively regulates seed germination and seedling development, further studies are required for identification of the downstream target genes of AtC3H12.

Collectively, our data propose that AtC3H12 containing three $\mathrm{CCCH}$ zinc finger motifs acts as a nuclear transcriptional activator to regulate the transcription of genes that negatively modulate seed germination and seedling development in Arabidopsis (Figure 9). 


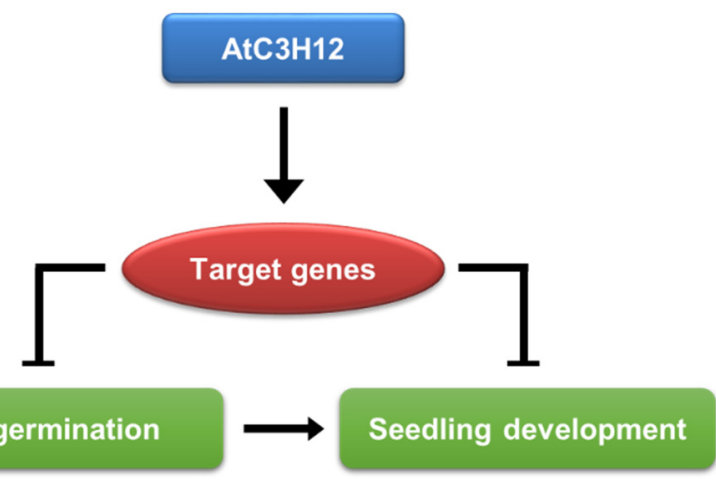

Figure 9. A functional model of the role of AtC3H12 in Arabidopsis development.

\section{Materials and Methods}

\subsection{Arabidopsis Growth}

Arabidopsis plants used in this research were of the Columbia (Col-0) ecotype. Arabidopsis seeds were prepared, germinated, and grown as previously described [23].

\subsection{Multiple-Sequence Alignment}

BLASTP analysis was conducted using NCBI BLAST (https:/ /blast.ncbi.nlm.nih.gov / Blast.cgi, accessed on 12 August 2020). ClustalW2 (https:/ / ebi.ac.uk/Tools/msa/clustalo, accessed on 12 August 2020) was used for multiple-sequence alignment.

\subsection{Plasmid Construction}

To generate constructs for subcellular localization, the full-length ORF of AtC3H12 was cloned into pFGL1283 and pFGL1292 in frame with N-terminal and C-terminal sGFP, respectively [36]. To clone constructs for GUS assay, a 282-bp upstream region from the transcriptional start site of $A t C 3 H 12$, with and without 559-bp 5' UTR, was fused to the GUS gene [6]. To generate vectors for AtC3H12 overexpression, the full-length ORF of AtC3H12 was inserted into pFGL1434, including the modified CaMV $35 S$ promoter and N-terminal-fused HA tag [36].

To clone constructs for transactivation activity analysis in yeast, full-length ORF and partial fragments of AtC3H12 were cloned into the pBD-GAL4 in frame with GAL4 BD. To generate vectors for the transactivation assay in Arabidopsis protoplasts, full-length ORF and partial fragments of AtC3H12 were fused to GAL4 BD under the control of the modified CaMV $35 S$ promoter [31].

Primers for cloning are shown in Table S1.

\subsection{Transgenic Plants and T-DNA-Inserted Mutants}

The constructs were transformed into Agrobacterium tumefaciens strain GV3101 (pMP90) using the freeze-thaw method [37] and then introduced into WT Arabidopsis using the floral-dipping method [38]. Transgenic plants were selected on MS agar plates containing kanamycin $(50 \mu \mathrm{g} / \mathrm{mL})$. The $\mathrm{T}_{3}$ homozygous lines were used for further experiments.

T-DNA-inserted atc3h12 mutant, SALK_011253 (atc3h12) was provided by the Salk Institute Genomic Analysis Laboratory.

\subsection{Protoplast Transformation}

The isolation and polyethylene glycol-mediated transformations of Arabidopsis protoplasts were conducted in accordance with Yoo et al. [39].

\subsection{Analysis of the Transactivation Activity in Yeast}

Yeast strain YD116 [40] was transformed using the Frozen-EZ Yeast Transformation II $^{\mathrm{TM}}$ Kit (Zymo Research Corp., Irvine, CA, USA), in accordance with the manufacturer's instructions. 
Quantitative $\beta$-galactosidase assay, $\beta$-galactosidase filter assay, and yeast growth assay were conducted as previously described [23]. In brief, a quantitative $\beta$-galactosidase activity using ONPG was quantified with the formula $1000 \times \mathrm{OD}_{420} /\left(\mathrm{OD}_{600} \times\right.$ assay time in $\mathrm{min} \times$ assay volume in $\mathrm{mL}$ ). $\beta$-Galactosidase filter assay was conducted using 5 -bromo4-chloro-3-indolyl- $\beta$-d-galactopyranoside as a substrate for $6 \mathrm{~h}$. Yeast transformants grown on SD media lacking Trp and Ura were incubated for $3-5$ days at $30{ }^{\circ} \mathrm{C}$ for the growth assay.

\subsection{Dual-luciferase Assay}

Firefly luciferase and Nano luciferase activities were quantified using the GloMax®Multi+ Detection System (Promega Corp., Madison, WI, USA) with Instinct ${ }^{\mathrm{TM}}$ Software and Nano-Glo®Dual-Luciferase®Reporter Assay System (Promega Corp., Madison, WI, USA).

\subsection{RNA Isolation and RT-PCR}

Total RNA was isolated using the RNAqueous RNA Isolation Kit (Invitrogen, Carlsbad, CA, USA) and Plant RNA Isolation Aid (Invitrogen, Carlsbad, CA, USA), in accordance with the manufacturer's protocol. Total RNA $(2 \mu \mathrm{g})$ was used for reverse transcription using Moloney murine leukemia virus reverse transcriptase (Promega Corp., Madison, WI, USA) as previously described [23].

RT-qPCR was conducted using a QuantStudio ${ }^{\mathrm{TM}} 3$ real-time PCR system (Applied Biosystems, Foster, CA, USA) and Power SYBR ${ }^{\mathrm{TM}}$ Green PCR Master Mix (Applied Biosystems, Foster, CA, USA) in accordance with manufacturer's manual. Real-time DNA amplification was analyzed using QuantStudio ${ }^{\mathrm{TM}}$ Design and Analysis software (version 1.4.3) (Applied Biosystems, Foster, CA, USA). Three independent reactions were conducted for each technical replicate. Two technical replicates were conducted for each biological replicate.

Semi-quantitative RT-PCR was conducted in accordance with previous study [23]. PCR reactions were repeated 30-31 cycles for AtC3H12 and 23-24 cycles for GAPc.

Primers for RT-PCR are shown in Table S2.

\subsection{GUS Assay}

Histochemical GUS assay was performed in accordance with the method described previously [23].

\subsection{Phenotype Analysis}

To measure the germination percentage, FW, and primary root length, 30 seeds of each plant were sown on the same MS agar plate and grown under SD conditions. Germination was determined by radicle protrusion. Primary root length was measured using ImageJ [41]. At least three biological replicates were performed.

\subsection{Statistical Analysis}

Statistical analysis was performed by IBM SPSS Statistics software version 23 (IBM Corp., Armonk, NY, USA) with one-way ANOVA using Tukey's multiple comparison test.

Supplementary Materials: The following supporting information can be downloaded at: https: //www.mdpi.com/article/10.3390/ijms23031572/s1.

Author Contributions: Conceptualization, H.-Y.S. and Y.-H.M.; methodology, T.K., H.-Y.S. and Y.H.M.; validation, T.K., H.-Y.S. and S.-Y.L.; investigation, T.K. and H.-Y.S.; writing-original draft preparation, T.K. and H.-Y.S. and S.-Y.L.; writing-review and editing, H.-Y.S., S.-Y.L. and Y.-H.M.; supervision, Y.-H.M.; project administration, Y.-H.M.; funding acquisition, Y.-H.M. All authors have read and agreed to the published version of the manuscript.

Funding: This work was supported by the National Research Foundation of Korea (NRF) grant funded by the Ministry of Education (No. 2020R1I1A3065749 and No. 2020R1I1A1A01065816). 
Institutional Review Board Statement: Not applicable.

Informed Consent Statement: Not applicable.

Data Availability Statement: The data presented in this study are available in the Supplementary Materials provided here.

Conflicts of Interest: The authors declare no conflict of interest.

\section{References}

1. Pomeranz, M.; Finer, J.; Jang, J.C. Putative molecular mechanisms underlying tandem CCCH zinc finger protein mediated plant growth, stress, and gene expression responses. Plant Signal. Behav. 2011, 6, 647-651. [CrossRef]

2. Ciftci-Yilmaz, S.; Mittler, R. The zinc finger network of plants. Cell. Mol. Life Sci. 2008, 65, 1150-1160. [CrossRef]

3. Laity, J.H.; Lee, B.M.; Wright, P.E. Zinc finger proteins: New insights into structural and functional diversity. Curr. Opin. Struct. Biol. 2001, 11, 39-46. [CrossRef]

4. Chai, G.; Kong, Y.; Zhu, M.; Yu, L.; Qi, G.; Tang, X.; Wang, Z.; Cao, Y.; Yu, C.; Zhou, G. Arabidopsis C3H14 and C3H15 have overlapping roles in the regulation of secondary wall thickening and anther development. J. Exp. Bot. 2015, 66, 2595-2609. [CrossRef] [PubMed]

5. Wang, D.; Guo, Y.; Wu, C.; Yang, G.; Li, Y.; Zheng, C. Genome-wide analysis of CCCH zinc finger family in Arabidopsis and rice. BMC Genom. 2008, 9, 44. [CrossRef] [PubMed]

6. Seok, H.Y.; Woo, D.H.; Park, H.Y.; Lee, S.Y.; Tran, H.T.; Lee, E.H.; Nguyen, L.V.; Moon, Y.H. AtC3H17, a non-tandem CCCH zinc finger protein, functions as a nuclear transcriptional activator and has pleiotropic effects on vegetative development, flowering and seed development in Arabidopsis. Plant Cell Physiol. 2016, 57, 603-615. [CrossRef] [PubMed]

7. Huang, P.; Ju, H.W.; Min, J.H.; Zhang, X.; Chung, J.S.; Cheong, H.S.; Kim, C.S. Molecular and physiological characterization of the Arabidopsis thaliana Oxidation-related Zinc Finger 2, a plasma membrane protein involved in ABA and salt stress response through the ABI2-mediated signaling pathway. Plant Cell Physiol. 2012, 53, 193-203. [CrossRef]

8. Kim, D.H.; Yamaguchi, S.; Lim, S.; Oh, E.; Park, J.; Hanada, A.; Kamiya, Y.; Choi, G. SOMNUS, a CCCH-type zinc finger protein in Arabidopsis, negatively regulates light-dependent seed germination downstream of PIL5. Plant Cell 2008, 20, $1260-1277$. [CrossRef]

9. Li, Z.; Thomas, T.L. PEI1, an embryo-specific zinc finger protein gene required for heart-stage embryo formation in Arabidopsis Plant Cell 1998, 10, 383-398. [CrossRef]

10. Maldonado-Bonilla, L.D.; Eschen-Lippold, L.; Gago-Zachert, S.; Tabassum, N.; Bauer, N.; Scheel, D.; Lee, J. The Arabidopsis tandem zinc finger 9 protein binds RNA and mediates pathogen-associated molecular pattern-triggered immune responses. Plant Cell Physiol. 2014, 55, 412-425. [CrossRef]

11. Sun, J.; Jiang, H.; Xu, Y.; Li, H.; Wu, X.; Xie, Q.; Li, C. The CCCH-type zinc finger proteins AtSZF1 and AtSZF2 regulate salt stress responses in Arabidopsis. Plant Cell Physiol. 2007, 48, 1148-1158. [CrossRef] [PubMed]

12. Jan, A.; Maruyama, K.; Todaka, D.; Kidokoro, S.; Abo, M.; Yoshimura, E.; Shinozaki, K.; Nakashima, K.; Yamaguchi-Shinozaki, K. OsTZF1, a CCCH-tandem zinc finger protein, confers delayed senescence and stress tolerance in rice by regulating stress-related genes. Plant Physiol. 2013, 161, 1202-1216. [CrossRef] [PubMed]

13. Seong, S.Y.; Shim, J.S.; Bang, S.W.; Kim, J.K. Overexpression of OsC3H10, a CCCH-zinc finger, improves drought tolerance in rice by regulating stress-related genes. Plants 2020, 9, 1298. [CrossRef] [PubMed]

14. Xie, Z.; Lin, W.; Yu, G.; Cheng, Q.; Xu, B.; Huang, B. Improved cold tolerance in switchgrass by a novel CCCH-type zinc finger transcription factor gene, PvC3H72, associated with ICE1-CBF-COR regulon and ABA-responsive genes. Biotechnol. Biofuels 2019, 12, 224. [CrossRef]

15. Chen, F.; Liu, H.L.; Wang, K.; Gao, Y.M.; Wu, M.; Xiang, Y. Identification of CCCH zinc finger proteins family in moso bamboo (Phyllostachys edulis), and PeC3H74 confers drought tolerance to transgenic plants. Front. Plant Sci. 2020, 11, 579255. [CrossRef]

16. Zhuang, Y.; Wang, C.; Zhang, Y.; Chen, S.; Wang, D.; Liu, Q.; Zhou, G.; Chai, G. Overexpression of PdC3H17 confers tolerance to drought stress depending on its CCCH domain in Populus. Front. Plant Sci. 2020, 10, 1748. [CrossRef]

17. Yan, Z.; Jia, J.; Yan, X.; Shi, H.; Han, Y. Arabidopsis KHZ1 and KHZ2, two novel non-tandem CCCH zinc-finger and K-homolog domain proteins, have redundant roles in the regulation of flowering and senescence. Plant Mol. Biol. 2017, 95, 549-565. [CrossRef]

18. Seok, H.Y.; Nguyen, L.V.; Park, H.Y.; Tarte, V.N.; Ha, J.; Lee, S.Y.; Moon, Y.H. Arabidopsis non-TZF gene AtC3H17 functions as a positive regulator in salt stress response. Biochem. Biophys. Res. Commun. 2018, 498, 954-959. [CrossRef]

19. Wang, L.; Xu, Y.; Zhang, C.; Ma, Q.; Joo, S.H.; Kim, S.K.; Xu, Z.; Chong, K. OsLIC, a novel CCCH-type zinc finger protein with transcription activation, mediates rice architecture via brassinosteroids signaling. PLoS ONE 2008, 3, e3521. [CrossRef]

20. Zhang, H.; Gao, X.; Zhi, Y.; Li, X.; Zhang, Q.; Niu, J.; Wang, J.; Zhai, H.; Zhao, N.; Li, J.; et al. A non-tandem CCCH-type zinc-finger protein, $\mathrm{IbC} 3 \mathrm{H} 18$, functions as a nuclear transcriptional activator and enhances abiotic stress tolerance in sweet potato. New Phytol. 2019, 223, 1918-1936. [CrossRef]

21. Xu, L.; Liu, T.; Xiong, X.; Liu, W.; Yu, Y.; Cao, J. Overexpression of two CCCH-type zinc-finger protein genes leads to pollen abortion in Brassica campestris ssp. chinensis. Genes 2020, 11, 1287. [CrossRef] [PubMed] 
22. Xu, L.; Xiong, X.; Liu, W.; Liu, T.; Yu, Y.; Cao, J. BcMF30a and BcMF30c, two novel non-tandem CCCH zinc finger proteins, function in pollen development and pollen germination in Brassica campestris ssp. chinensis. Int. J. Mol. Sci. 2020, 21, 6428. [CrossRef] [PubMed]

23. Seok, H.Y.; Bae, H.; Kim, T.; Mehdi, S.M.M.; Nguyen, L.V.; Lee, S.Y.; Moon, Y.H. Non-TZF gene protein AtC3H59/ZFWD3 is involved in seed germination, seedling development, and seed development, interacting with PPPDE family protein Desi1 in Arabidopsis. Int. J. Mol. Sci. 2021, 22, 4738. [CrossRef]

24. Li, J.; Jia, D.; Chen, X. HUA1, a regulator of stamen and carpel identities in Arabidopsis, codes for a nuclear RNA binding protein. Plant Cell 2001, 13, 2269-2281. [CrossRef] [PubMed]

25. Pomeranz, M.C.; Hah, C.; Lin, P.C.; Kang, S.G.; Finer, J.J.; Blackshear, P.J.; Jang, J.C. The Arabidopsis tandem zinc finger protein AtTZF1 traffics between the nucleus and cytoplasmic foci and binds both DNA and RNA. Plant Physiol. 2010, 152, 151-165. [CrossRef] [PubMed]

26. Hunt, A.G. The Arabidopsis polyadenylation factor subunit CPSF30 as conceptual link between mRNA polyadenylation and cellular signaling. Curr. Opin. Plant Biol. 2014, 21, 128-132. [CrossRef] [PubMed]

27. Addepalli, B.; Hunt, A.G. Ribonuclease activity is a common property of Arabidopsis CCCH-containing zinc-finger proteins. FEBS Lett. 2008, 582, 2577-2582. [CrossRef]

28. Capella, M.; Re, D.A.; Arce, A.L.; Chan, R.L. Plant homeodomain-leucine zipper I transcription factors exhibit different functional AHA motifs that selectively interact with TBP or/and TFIIB. Plant Cell Rep. 2014, 33, 955-967. [CrossRef]

29. Tiwari, S.B.; Belachew, A.; Ma, S.F.; Young, M.; Ade, J.; Shen, Y.; Marion, C.M.; Holtan, H.E.; Bailey, A.; Stone, J.K.; et al. The EDLL motif: A potent plant transcriptional activation domain from AP2/ERF transcription factors. Plant J. 2012, 70, 855-865. [CrossRef]

30. Bui, L.T.; Giuntoli, B.; Kosmacz, M.; Parlanti, S.; Licausi, F. Constitutively expressed ERF-VII transcription factors redundantly activate the core anaerobic response in Arabidopsis thaliana. Plant Sci. 2015, 236, 37-43. [CrossRef]

31. Seok, H.Y.; Ha, J.; Lee, S.Y.; Bae, H.; Moon, Y.H. Two alternative splicing variants of AtERF73/HRE1, HRE1 $\alpha$ and HRE1 $\beta$, have differential transactivation activities in Arabidopsis. Int. J. Mol. Sci. 2020, 21, 6984. [CrossRef] [PubMed]

32. Chou, C.C.; Wang, A.H. Structural D/E-rich repeats play multiple roles especially in gene regulation through DNA/RNA mimicry. Mol. Biosyst. 2015, 11, 2144-2151. [CrossRef] [PubMed]

33. Kim, W.C.; Kim, J.Y.; Ko, J.H.; Kang, H.; Kim, J.; Han, K.H. AtC3H14, a plant-specific tandem CCCH zinc-finger protein, binds to its target mRNAs in a sequence-specific manner and affects cell elongation in Arabidopsis thaliana. Plant J. 2014, 80, 772-784. [CrossRef] [PubMed]

34. Zhang, C.; Xu, Y.; Guo, S.; Zhu, J.; Huan, Q.; Liu, H.; Wang, L.; Luo, G.; Wang, X.; Chong, K. Dynamics of brassinosteroids response modulated by negative regulator LIC in rice. PLoS Genet. 2012, 8, e1002686. [CrossRef]

35. Stetter, M.G.; Schmid, K.; Ludewig, U. Uncovering genes and ploidy involved in the high diversity in root hair density, length and response to local scarce phosphate in Arabidopsis thaliana. PLoS ONE 2015, 10, e0120604. [CrossRef] [PubMed]

36. Nguyen, L.V.; Seok, H.Y.; Woo, D.H.; Lee, S.Y.; Moon, Y.H. Overexpression of the DEAD-box RNA helicase gene AtRH17 confers tolerance to salt stress in Arabidopsis. Int. J. Mol. Sci. 2018, 19, 3777. [CrossRef] [PubMed]

37. Hofgen, R.; Willmitzer, L. Storage of competent cells for Agrobacterium transformation. Nucleic Acids Res. 1988, 16, 9877. [CrossRef] [PubMed]

38. Clough, S.J.; Bent, A.F. Floral dip: A simplified method for Agrobacterium-mediated transformation of Arabidopsis thaliana. Plant J. 1998, 16, 735-743. [CrossRef] [PubMed]

39. Yoo, S.D.; Cho, Y.H.; Sheen, J. Arabidopsis mesophyll protoplasts: A versatile cell system for transient gene expression analysis. Nat. Protoc. 2007, 2, 1565-1572. [CrossRef] [PubMed]

40. Cid, V.J.; Shulewitz, M.J.; McDonald, K.L.; Thorner, J. Dynamic localization of the Swe1 regulator Hsl7 during the Saccharomyces cerevisiae cell cycle. Mol. Biol. Cell 2001, 12, 1645-1669. [CrossRef]

41. Schneider, C.A.; Rasband, W.S.; Eliceiri, K.W. NIH Image to ImageJ: 25 years of image analysis. Nat. Methods 2012, 9, 671-675. [CrossRef] [PubMed] 